\title{
e-Migrinter
}

15 | 2017

Migrinter a trente ans : analyses et portraits

\section{Autonomie et projet migratoire : deux notions abordées à travers les travaux d'Emmanuel Ma Mung}

Iris Polyzos

\section{(2) OpenEdition}

\section{Journals}

Édition électronique

URL : https://journals.openedition.org/e-migrinter/822

DOI : 10.4000/e-migrinter.822

ISSN : 1961-9685

Éditeur

UMR 7301 - Migrinter

Référence électronique

Iris Polyzos, «Autonomie et projet migratoire : deux notions abordées à travers les travaux d'Emmanuel Ma Mung », e-Migrinter [En ligne], 15 | 2017, mis en ligne le 09 mai 2017, consulté le 20 mai 2021. URL : http://journals.openedition.org/e-migrinter/822 ; DOI : https://doi.org/10.4000/emigrinter.822

Ce document a été généré automatiquement le 20 mai 2021.

Tous droits réservés 


\section{Autonomie et projet migratoire : deux notions abordées à travers les travaux d'Emmanuel Ma Mung}

Iris Polyzos

\section{Introduction}

Parmi ses publications, quatre textes caractéristiques ont été choisis pour être étudiés dans cet article : les deux premiers analysent les recompositions des villes de Sbiba et M'saken en Tunisie et introduisent la notion d'autonomie $(1983 ; 1986)$. Le troisième texte est l'Habilitation à Diriger des Recherches (HDR) de l'auteur, dans laquelle il propose une définition analytique de cette notion (1999). Enfin, le quatrième discute plus en profondeur la notion de projet migratoire (2009). Dans le but de souligner les liens qui se tissent entre production académique et parcours personnel de l'auteur, l'article s'appuie également sur un entretien inédit réalisé en décembre 2015 avec Emmanuel Ma Mung. Cet entretien s'articule sur cinq questions ouvertes qui mettent en avant les principales influences théoriques qui ont permis à l'auteur de développer les notions d'autonomie et de projet migratoire.

2 Avant de présenter brièvement les notions qui nous intéressent, il faut d'abord clarifier le fait que ces notions apparaissent de façon distincte dans les travaux du géographe. En effet, la notion d'autonomie du sujet occupe une place majeure tout au long de la réflexion de l'auteur, tandis que l'utilisation de la notion du projet migratoire arrive plus tardivement et ne concerne que la thématique des migrations internationales. 


\section{Évolution des notions d'autonomie et de projet migratoire abordées à travers ses principaux travaux de recherche}

3 Les premières influences qui forment la réflexion d'Emmanuel Ma Mung autour du concept d'autonomie sont d'ordres politiques et philosophiques. Elles marquent l'auteur, ainsi qu'une partie de la génération des années 1960. Emmanuel Ma Mung, jeune lycéen à l'époque, vient au contact des grands textes de l'idéologie anarchiste qui mettent fortement en cause les logiques de domination par l'État. Ce courant politique va clairement influencer ses travaux autour de la notion d'autonomie, une notion qui se fait jour dès ses premiers travaux scientifiques.

Dans les années 1980, le choix de son premier objet de recherche dans le cadre d'un Diplôme d'Études Approfondies (DEA) de géographie, ainsi que pour l'obtention d'un doctorat dans le même domaine, concerne les relations d'une société agraire des hautes steppes tunisiennes face à l'État. L'étude de la ville de Sbiba et de ses recompositions sociales et économiques lui permet de montrer les logiques de différenciation de cette entité sociale vis-à-vis du pouvoir étatique $(1980 ; 1983)$. En outre, à travers l'étude de la ville de M'saken en Tunisie, réalisée dans le cadre d'un programme de recherche menée à Migrinter, l'auteur étudie le rôle des migrations dans les recompositions des sociétés locales. Dans cette ville en transition, les émigrants des années 1960-1970 apparaissent comme des acteurs du changement, puisque la mobilisation des transferts migratoires dans certains secteurs économiques, comme le marché foncier ou le commerce, ont un véritable impact à l'échelle locale. Ainsi, Emmanuel Ma Mung souligne que " l'émigré n'apparaît plus comme un simple pourvoyeur de fonds mais devient un opérateur financier dans le sens où il maitrise son épargne en vue de réaliser des profits » $(1986: 167)$.

5 Au début des années 1990, le commerce tenu par des populations étrangères en France devient pour lui un nouveau champ d'étude qui lui permettra d'affiner le « point de vue de l'autonomie » (1999: 13). Il rédige de nombreux articles sur l'entreprenariat des migrants maghrébins et chinois, établis en France et notamment dans l'agglomération parisienne. Les "initiatives économiques" (1999: 137) et les "territoires marchands » qui se développent à l'échelle locale (1999: 212) vont alimenter sa réflexion. Dans sa thèse de HDR il explique, d'une façon analytique, que le commerçant migrant se situe dans une relation de distance et de proximité tant vis-à-vis de la société d'accueil, qu'envers son propre groupe migratoire. Cette dynamique de différentiation introduit l'individu dans un processus de recomposition de son identité.

Durant cette même période, les recherches sur le commerce ethnique conduisent Emmanuel Ma Mung à questionner plus en profondeur les diasporas et leur dimension spatiale. Le cas de la diaspora chinoise et de ses implantations urbaines à Paris devient la principale étude de cas élaborée dans son ouvrage La diaspora chinoise. Géographie d'une migration, publié en 2000. Les logiques d'organisation d'une diaspora comme entité sociale qui, d'une part, maintient des distances avec la société de départ et, d'autre part, se trouve en contact proche avec celle-ci, renvoient à la notion d'autonomie. L'autonomie s'exprime à travers la possibilité des diasporas à se reproduire dans et à travers leur dispersion. 
7 Le projet migratoire, deuxième notion discutée par le géographe, est surtout analysé dans l'article "Le point de vue de l'autonomie dans l'étude des migrations internationales: "penser de l'intérieur" les phénomènes de mobilité " (2009). Après une première présentation d'autres études discutant de la notion de projet migratoire, réalisées surtout par des historiens (Rosental, 1999) ou des géographes (Boyer, 2005), l'auteur souligne que le projet migratoire constitue plutôt une approche épistémologique dans l'étude des mobilités et moins une " entité observable » ou une notion explicitement définie. Il permet la compréhension des mobilités au-delà des facteurs strictement économiques ou structurels et "indique une position de l'observateur " (2009: 32) vis-à-vis de son objet de recherche: une position qui met surtout en avant les migrants en tant qu'acteurs de leur propre parcours. Plus précisément, l'auteur souligne que l'essentiel est de comprendre les migrants à travers leur capacité d'agir sur les normes et les structures du contexte d'accueil, sur lesdites "dispositions extérieures ". Il propose alors le terme de "création des mondes migratoires » (2009) qui permet de mieux étudier la manière dont le migrant imagine et réalise ce mouvement, en tenant compte des contraintes et des opportunités qui caractérisent le contexte de départ et d'arrivée.

\section{Le point de vue de l'auteur}

8 Cette partie de l'article reprend cinq questions que nous avons adressées à Emmanuel Ma Mung lors de notre entretien. Elles essaient de mettre en avant les interrelations entre les principales lectures qui l'ont influencé et l'évolution des notions d'autonomie et de projet migratoire.

1/ Comment votre parcours personnel a-t-il influencé vos réflexions sur les notions d'autonomie et de projet migratoire?

En ce qui concerne l'autonomie, cela a été d'abord pour moi une question politique qui date de mes années lycéennes, liée à mes orientations libertaires. À cette époquelà (fin des années 1960) mes copains de lycée et moi lisions les classiques anarchistes : Pierre-Joseph Proudhon, Michel Bakounine, Daniel Guérin mais aussi Voline (La révolution inconnue), et Elisée Reclus. Nous étions libertaires et antiétatiques, et aussi opposés aux mouvements marxistes-léninistes qui florissaient à l'époque. Nous étions aussi attirés par la mouvance situationniste. Et des exemplaires de la revue de l'Internationale Situationniste (IS) circulaient entre nous, ainsi que le bouquin Traité de savoir-vivre à l'usage des jeunes générations de Raoul Vaneigem et La société du spectacle de Guy Debord, livre auquel les adolescents que nous étions ne comprenaient pas toujours grand-chose. C'est sans doute aussi durant cette période lycéenne que j'ai commencé à m'intéresser à la géographie (même si plus tard j'ai commencé par faire une licence d'histoire avant celle de géographie). D'abord à cause d'Elisée Reclus que je connaissais avant tout comme " théoricien » anarchiste et un des animateurs de la Commune de Paris où il avait tenté de mettre en œuvre des principes libertaires. Le fait qu'il soit géographe et qu'il présente cette matière comme propre à libérer l'être humain grâce à la connaissance qu'elle donne du monde m'en a donné une vision très positive. Une autre raison est que j'avais vu dans un numéro de la revue de l'IS une carte qui m'avait surpris et intrigué et qui représentait les déplacements d'une étudiante du $16^{\text {ème }}$ arrondissement de Paris, carte que, si je me souviens bien, l'IS présentait dans le cadre de la « psychogéographie » qu'elle souhaitait promouvoir. 
Cela me rappelle aussi que Henri Lefebvre était lié aux « situs » comme on disait à l'époque ${ }^{1}$.

Pour revenir à l'autonomie, c'est cette question que j'ai continuée à me poser lorsque j'ai commencé l'université. Mais il ne faut pas croire qu'un jour je me suis dit : « bon, à partir de maintenant je vais étudier l'autonomie ». Et encore moins: " je vais développer une approche théorique basée sur l'autonomie ». Les choses ne se sont pas passées ainsi. La question s'est peu à peu imposée sans que je m'en rende vraiment compte. Comme je le dirai plus tard, c'est lorsque j'ai préparé mon HDR et que je me suis interrogé sur ce qui reliait mes différents travaux que je me suis aperçu - et que j'ai même été surpris - qu'ils étaient reliés par une interrogation sur l'autonomie. Il y avait un côté un peu obsessionnel dans tout cela. Edgar Morin parle à propos de certains thèmes de recherche "d'obsessions cognitives ", c'est bien de cela dont il s'agit ici. La notion de projet migratoire est venue beaucoup plus tard. J'expliquerai cela tout à l'heure.

2/ Comment ces deux notions ont-elles évolué des premiers travaux jusqu'à aujourd'hui ?

Ma maîtrise de géographie, que j'ai faite avec Yves Lacoste en 1977, s'intitulait Intervention de l'État et hiérarchisation de l'espace agricole, le cas de Sbiba, hautes steppes tunisiennes. Le titre était tout à fait explicite et mon but était de montrer comment l'État tunisien avait contribué à fragmenter la société locale en imposant une réforme agraire sans demander leur avis aux gens et même contre leur volonté. C'est la même problématique des rapports conflictuels entre la société et l'État que j'ai approfondie dans ma thèse. J'essayais d'y formuler une conception de l'autonomie comprise comme autonomie sociale au sens d'autonomie de la société vis-à-vis de l'État. Il n'y avait pas de problématique migratoire au sens où on l'entend aujourd'hui, même si la société des hautes steppes tunisiennes avait été dans le passé une société pastorale semi-nomade. Et je ne parlais pas de projet migratoire car ce n'était pas mon sujet dans cette thèse.

C'est lorsque j'ai étudié les migrations internationales que j'ai parlé de projet migratoire, assez tardivement d'ailleurs, car j'avais des hésitations à utiliser l'expression. Je trouvais qu'elle connotait trop l'idée d'un migrant entièrement libre de ses choix et qui ressemblait ainsi à l'individu totalement fictif des idéologues du marché soi-disant libre. C'est d'ailleurs le même problème avec la notion d'autonomie qui a été détournée, " récupérée " aurait-on dit dans ma jeunesse, par l'idéologie contemporaine pseudo-libérale. J'y reviendrai peut-être tout à l'heure. A posteriori, il me semble plutôt qu'à l'époque je ne posais pas l'autonomie comme notion permettant d'appréhender les situations que j'étudiais. Je dirais plutôt que, consciemment ou non, j'essayais de les aborder dans une perspective générale visant l'autonomie.

Restait à définir l'autonomie. Je ne l'ai pas fait, ni tenté de le faire avant mon HDR. Et je l'ai fait à partir de mes travaux en me demandant comment l'autonomie se manifestait dans les situations que j'étudiais et ce qu'on pouvait en dire à partir de cela. En résumant, on pourrait dire, comme je l'ai fait dans un article (2009), que distinction et différenciation d'une part, affirmation et initiative de l'autre, sont les deux ensembles de sens que nous pouvons donner à l'autonomie. Ils ne correspondent pas à deux aspects séparés de celle-ci, ils en décrivent plutôt le double mouvement, la double forme qu'elle prend simultanément. Cette double forme 
s'exprime dans l'action et la pratique par un savoir-faire comme capacité de distinction et de différenciation du sujet (individuel ou collectif) et de constitution d'un monde et par un pouvoir-faire comme capacité d'affirmation et d'initiative du sujet et de transformation de ce monde.

Comme je le disais tout à l'heure, lorsque j'ai préparé mon HDR à la fin des années 1990 , je me suis posé la question de savoir ce qui reliait des recherches aussi différentes que la société des hautes steppes tunisiennes, le commerce ethnique, les transferts migratoires, les diasporas, etc. J'ai été surpris de constater que ce qui les reliait était une interrogation sur l'autonomie.

L'autonomie n'est pas un concept dont on pourrait apprécier les contours et les contenus et qu'on pourrait ensuite confronter au réel pour voir dans celui-ci ce qui résulte de l'autonomie et ce qui n'en résulte pas. L'autonomie se construit, à proprement parler, aussi bien dans la chose observée que du point de vue de l'observateur. C'est pour cela que j'ai des réticences avec l'agentivité ou agency. Dans cette approche, l'autonomie des sujets est un donné qu'on peut aller observer. Elle existe à l'état de denrée naturelle chez les individus, elle est le résultat direct de leur capacité d'action (je caricature bien entendu). Pour moi, l'autonomie est une visée qui la construit. Autrement dit, viser l'autonomie c'est déjà l'établir (ou du moins établir ses prémices). La dimension politique est première. Il y a quelque chose de circulaire dans l'autonomie. D'ailleurs Vincent Descombes (2004) parle du cercle de l'autonomie. Mais pour moi, ce cercle est plutôt une spirale, un cercle qui avance. Sinon ce serait la reproduction ininterrompue du même.

Au cours de mes recherches ultérieures, j'ai toujours eu en arrière-plan la perspective de l'autonomie, ce qui a défini, par "construction " pour ainsi dire, une épistémologie qu'il serait trop long d'expliciter ici. Par ailleurs, il y a un point sur lequel j'insiste toujours, c'est que l'autonomie n'est pas l'autarcie. C'en est même le contraire. De même, autonomie ne signifie pas absence de contraintes, bien au contraire, car s'il y a pouvoir-faire du sujet - au sens d'initiative - c'est qu'il y a aussi contraintes, et l'on sait qu'elles peuvent être très importantes pour les migrants. Le pouvoir-faire c'est la capacité de transformer ces contraintes en un « pour-soi » du sujet.

Aujourd'hui, j'essaie de relier la perspective de l'autonomie développée dans mes travaux sur les migrations internationales à celle que j'ai mise à l'épreuve dans mon essai sur l'espace géographique qui date de 1999 (que je n'ai malheureusement pas pris le soin de publier) et qui constitue la troisième partie de mon HDR.

3/ Quelles sont les principales lectures qui ont influencé vos réflexions?

Hormis Yves Lacoste qui a dirigé ma maîtrise et mon DEA de géographie, j'avoue que je n'ai pas été marqué par les géographes - excepté Élisée Reclus, mais je n'ai jamais vraiment utilisé ses travaux. Les auteurs qui m'ont le plus marqué dans mes recherches sont, par ordre chronologique, Pierre Clastres, Cornélius Castoriadis, Edgar Morin et Francisco Varela, biologiste chilien qui a écrit notamment Autonomie et connaissance, essai sur le vivant (1989).

Lorsque je faisais ma thèse sur les hautes steppes tunisiennes à la fin des années 1970, j'ai beaucoup été marqué par les travaux d'un anthropologue, Pierre Clastres - qui pourtant étudiait des populations complètement différentes (les indiens Guayakis en Amazonie) - notamment par son livre La société contre l'État (1974) et un long article 
paru dans la revue Libre et qui s'intitulait « Archéologie de la violence » (1977a), ainsi que par " Malheur du guerrier sauvage " paru dans la même revue (1977b). Pierre Clastres a beaucoup marqué mon travail de thèse car il m'a fourni les éléments pour comprendre la société des hautes steppes tunisiennes et sa dissidence permanente vis-à-vis des pouvoirs centraux, que ce soit avant, pendant, ou après la colonisation. Pour Pierre Clastres, les sociétés archaïques ne sont pas " sans État ", [soit] dans une situation de sous-développement politique qui empêcherait l'apparition de l'État. Elles sont contre l'État et organisées pour en empêcher l'émergence. Comment et de quelle manière? Il répond à cela dans son article "Archéologie de la violence ». Ce qui caractérise ces sociétés, c'est qu'elles sont avant tout des sociétés guerrières dans lesquelles il y a une propriété collective de la violence. Il n'y a pas, comme dans les sociétés à État, un corps spécialisé dans l'usage de la violence (armée, police). C'est la propriété collective de la violence qui empêche l'apparition de ce corps spécialisé qui monopolise alors l'usage de la violence et qui préfigure l'État. Mais comme le souligne Pierre Clastres, la propriété collective de la violence n'implique pas la guerre permanente (car les gens ne passent pas tout leur temps à guerroyer), mais seulement la possibilité permanente de la guerre.

C'est à partir de cette grille d'analyse que j'ai étudié la société des hautes steppes tunisiennes. Et je pense que cela a bien fonctionné et permis une analyse originale de cette société, analyse à distance des théories marxistes tiers-mondistes ou anticolonialistes qui dominaient alors. Je dois dire que j'en ai bavé pendant ma soutenance, car le président du jury était Jean Dresh. Un homme remarquable, anticolonialiste de la première heure dès les années 1920 qui était furieux car ma thèse soutenait qu'il y avait une continuité entre la période précoloniale, la colonisation et l'indépendance, ce qui allait évidemment à contre-courant de ce qu'il pensait.

C'est dans les années 1970 et surtout 1980 que j'ai commencé à lire Cornelius Castoriadis. D'abord L'institution imaginaire de la société (1975) que l'étudiant que j'étais au moment de sa parution en 1975 avait bien du mal à assimiler vu la profondeur (et la taille) de l'ouvrage. Et ensuite, les six volumes des "Carrefours du labyrinthe ». Comme dit Edgar Morin, Cornelius Castoriadis est un " titan de la pensée ». Et la sienne m'a tellement imprégné que je ne saurais faire l'inventaire de ce que l'on retrouve de lui dans mes travaux. Mais je ne suis pas pour autant un " disciple " de celui-ci. Ce serait d'ailleurs contradictoire avec la volonté de développer une pensée autonome. La grande force des penseurs de l'autonomie que sont Cornelius Castoriadis, Pierre Clastres, Edgar Morin ou encore Francisco Varela et d'autres encore, c'est qu'ils nous apprennent à penser de façon autonome. C'est aussi ce que m'a permis de faire Edgar Morin, notamment avec ses six volumes de La Méthode $(1977,1980,1986,1991,2001,2004)$ qui traitent de façon tout à fait explicite dans certains chapitres de l'autonomie.

Leurs livres sont sans doute ceux que je relis le plus souvent, avec d'autres comme Vincent Descombes. La lecture de certains passages d'Edgard Morin, et surtout de Francisco Varela, m'a orienté vers la question de l'articulation de l'autonomie sociale/politique et de l'autonomie physique/biologique (autonomie du vivant), question que je me pose depuis quelques années et sur laquelle je peine, car il y a un risque évident de naturalisation de l'autonomie et de déterminisme biologique du politique. Je pense que c'est la notion de mobilité comme capacité à se déplacer qui 
permet de le faire. Or, l'une des formes de la mobilité, c'est la migration. Et là, je retombe sur mes pieds. C'est là aussi qu'intervient la notion de projet (migratoire), car projet veut dire aussi étymologiquement « se jeter en avant ». Il y donc là aussi de la mobilité physique.

\section{4/ Autonomie et projet migratoire : comment ces deux notions sont-t-elles liées ?}

- Est-ce l'approche théorique de l'autonomie qui a formé la notion de projet migratoire ou, à l'inverse, les réalités observées et donc le projet migratoire, qui ont nourri vos réflexions sur l'autonomie?

La question de l'autonomie est première, mais comme vous avez pu le voir tout à l'heure, elle n'était pas posée à propos des migrations internationales, mais de la société des hautes steppes tunisiennes.

Celle de projet migratoire est venue bien après. Elle s'est imposée progressivement, assez lentement, car j'avais un certain nombre de réticences à utiliser l'idée de projet migratoire. En effet, quand on parle de projet (comme lorsqu'on parle d'autonomie), on est tout de suite confronté à l'utilisation qu'en fait toute l'idéologie liée au marketing et au management des ressources humaines (la culture du projet, le travail par projet, en réseau, etc.) et au sens qu'elle donne à ces mots, sens qui est à l'opposé de la conception que je m'en fais. Tout ce qui se dit aujourd'hui à propos de la "culture du projet " et de l'autonomie dans le travail est un formidable détournement et une récupération par les institutions capitalistes du sens libérateur que ces mots ou leurs synonymes peuvent avoir. C'est ce que montrent très bien Luc Boltanski et Eve Chapiello dans " Le nouvel esprit du capitalisme " paru en 1999, ou, dans un registre différent, le sociologue Jean-Pierre Le Goff dans " La barbarie douce ", ouvrage paru lui aussi en 1999. J'ai commencé à parler de projet migratoire, non pas directement dans mes recherches, mais dans le rapport d'activité et le " projet " de recherche de Migrinter en 1995, dont j'étais responsable à l'époque. Puis, dans mon HDR. Je n'ai vraiment commencé à y réfléchir que dans le chapitre de l'ouvrage collectif dirigé par Françoise Dureau et Marie-Antoinette Hily Les mondes de la mobilité puis, dans le cadre des différentes séances du séminaire organisé par Migrinter sur ce thème.

- Si le projet migratoire est l'une des expressions de l'autonomie du migrant, peut-ont voir d'autres expressions de ce point de vue dans les migrations contemporaines?

Il y a par exemple le thème de l'entreprenariat ethnique. En évitant deux écueils : celui de l'idéologie entrepreneuriale et celui du culturalisme. Il y a aussi celui des mondes migratoires, c'est-à-dire la constitution de mondes-propres aux migrants, de mondes appropriés au sens que leur donne Henri Lefebvre (2000) (construction d'espace approprié à un groupe social plutôt qu'approprié par un groupe social). Et sans doute bien d'autres qui ne me viennent pas en tête maintenant.

5/ Quel est le rôle du contexte scientifique du laboratoire Migrinter dans l'évolution de ces notions?

L'autonomie en tant que telle n'est pas explicitement travaillée au sein du laboratoire. Cependant, plusieurs recherches ont recours directement ou indirectement à cette notion. Par contre, des travaux plus nombreux - et notamment des thèses - font appel à la notion de projet migratoire. Ces recherches ont nourri ma réflexion sur l'autonomie et le projet migratoire. Mais c'est surtout les séminaires et ateliers organisés au sein du laboratoire qui m'ont aidé à clarifier ces notions, notamment ceux consacrés aux mondes de la mobilité au milieu des années 2000, aux 
territoires ethniques marchands en 2007, aux expériences migratoires et au projet migratoire plus récemment. Les discussions et les questions, les présentations que l'on y fait obligent à des efforts d'explicitation qui m'ont beaucoup aidé. Notamment pour approfondir le lien entre autonomie et migration et plus profondément entre autonomie sociale et autonomie physique/biologique.

\section{Conclusion}

9 Les textes d'Emmanuel Ma Mung qui ont été analysés dans cet article montrent l'évolution diachronique des objets de recherche et des questionnements théoriques et politiques qui ont préoccupé l'auteur dès les débuts de son parcours: de la société tunisienne en transition au fonctionnement de l'entreprenariat ethnique ou encore aux mobilités contemporaines de la diaspora chinoise, l'auteur a élaboré la notion d'autonomie, tant comme " point de vue » du chercheur face à son objet de recherche, que comme logique de fonctionnement des groupes sociaux vis-à-vis des conditions extérieures qui leur sont imposées. La lecture de ces textes, publiés entre 1980 et 2010 (1983, 1986, 1999 et 2009), a été enrichie par un entretien qui a mis en avant les principales influences de l'auteur. En définitive, nous observons que, pour Emmanuel Ma Mung, la notion d'autonomie et du projet migratoire évoluent tant en fonction de ses objets de recherche, qu'en fonction des lectures ou relectures des principaux travaux qu'il cite durant l'entretien.

\section{BIBLIOGRAPHIE}

Boyer, Florence (2005) Le projet migratoire des migrants touaregs de la zone de Bankilaré : la pauvreté désavouée, Stichproben, $\mathrm{n}^{\circ}$ 8, pp. 47-67.

Ma Mung, Emmanuel (1980) Pouvoir et organisation de l'espace, Sbiba, Hautes Steppes tunisiennes, Paris, Université Paris VIII-Vincennes, 360 p., Th. Doct : Géogr. : Paris : 1980.

Ma Mung, Emmanuel (1983) Hiérarchisation de l'espace et intervention de l'État: Sbiba, hautes steppes tunisiennes, Études Méditerranéennes, Fascicule 5, pp. 85-127.

Ma Mung, Emmanuel (1986) L'impact des transferts migratoires dans la ville de M'saken (Tunisie), Revue Européenne des Migrations Internationales ; Méditerranée, vol. 2, n 1, pp. 163-178.

Ma Mung, Emmanuel ; Simon, Gildas (1990) Commerçants maghrébins et asiatiques en France, Paris, Masson, 138 p. (Recherches en Géographie).

Ma Mung, Emmanuel (1992) L'expansion du commerce ethnique : Asiatiques et Maghrébins dans la région parisienne, Revue Européenne des Migrations Internationales, vol. 8, nº 1, pp. 39-59.

Ma Mung, Emmanuel (1999) Autonomie, Migrations et Altérité, Poitiers, Université de Poitiers, 459 p., Habilitation à Diriger des Recherches : Géogr : Poitiers : 1999.

Ma Mung, Emmanuel (2000) La diaspora chinoise. Géographie d'une migration, Paris, Orphys, 175 p. 
Ma Mung, Emmanuel (2009) Le point de vue de l'autonomie dans l'étude des migrations internationales : "penser de l'intérieur" les phénomènes de mobilité, in Dureau, F. ; Hily, M.-A. (dir.) Les mondes de la mobilité, Rennes, Presses universitaires de Rennes, pp. 25-38.

Rosental, Paul-André (1999) Les sentiers invisibles. Espace, familles et migrations dans la France du 19ème siècle, Paris, Éditions de l'École des Hautes Études en Sciences Sociales, 255 p.

Références bibliographiques mentionnées par Emmanuel Ma Mung durant l'entretien. Boltanski, Luc ; Chiapello, Eve (1999) Le nouvel esprit du capitalisme, Paris, Gallimard, 843 p. Le Goff, Jean Pierre (2003) La barbarie douce : la modernisation aveugle des entreprises et de l'école, (nouvelle édition), Paris, La Découverte, $144 \mathrm{p}$.

Clastres, Pierre (1974) La société contre l'État, Paris, Éditions de Minuit, 186 p.

Clastres, Pierre (1977a) Archéologie de la violence, Libre, n 1, pp. 137-173.

Clastres, Pierre (1977b) Malheur du guerrier sauvage, Libre, $\mathrm{n}^{\circ}$ 2, pp. 69-109.

Castoriadis, Cornélius (1975) L'institution imaginaire de la société, Paris, Seuil, 502 p.

Castoriadis, Cornélius (1978) Les carrefours du labyrinthe (I), Paris, Seuil, 315 p.

Castoriadis, Cornélius (1986) Domaine de l'homme, les carrefours du labyrinthe (II), Paris, Seuil, 570 p.

Castoriadis, Cornélius (1990) Le monde morcelé, les carrefours du labyrinthe (III), Paris, Seuil, 277 p.

Castoriadis, Cornélius (1996) La montée de l'insignifiance, les carrefours du labyrinthe (IV), Paris, Seuil, $240 \mathrm{p}$.

Castoriadis, Cornélius (1997) Fait et à faire, les carrefours du labyrinthe (V), Paris, Seuil, 280 p.

Descombes, Vincent (1989) Philosophie par gros temps, Paris, Éditions de Minuit, 187 p.

Descombes, Vincent (1995) La denrée mentale, Paris, Éditions de Minuit, 348 p.

Descombes, Vincent (2004) Le complément de sujet, enquête sur le fait d'agir de soi-même, Paris, Gallimard, 521 p.

Descombes, Vincent (2007) Le raisonnement de l'ours, Paris, Le Seuil, 456 p.

Lefebvre, Henri (2000) La production de l'espace, (4ème édition), Paris, Anthropos, 487 p.

Morin, Edgar (1977) La méthode, 1, La nature de la nature, Paris, Seuil, 399 p.

Morin, Edgar (1980) La méthode, 2, La vie de la vie, Paris, Seuil, 420 p.

Morin, Edgar (1986) La méthode, 3, La connaissance de la connaissance, Paris, Seuil, 249 p.

Morin, Edgar (1991) La méthode, 4, Les idées, leur habitat, leur vie, leurs mœurs, leur organisation, Paris, Seuil, $262 \mathrm{p}$.

Morin, Edgar (2001) La méthode, 5, L’humanité de l'humanité, Paris, Seuil, 357 p.

Morin, Edgar (2004) La méthode, 6, Ethique, Paris, Seuil, 241 p.

Varela, Fransisco (1989) Autonomie et connaissance, Paris, Seuil, 250 p.

Varela, Fransisco (1989) Autonomie et connaissance : essai sur le vivant, Paris, Seuil, 247 p.

Varela, Fransisco ; Evan, Thompson ; Rosch, Eléanor (1993) L'inscription corporelle de l'esprit, Paris, Seuil, $377 \mathrm{p}$. 


\section{NOTES}

1. L'auteur se réfère aux situationnistes.

\section{RÉSUMÉS}

L'article s'articule autour de deux notions-clés pour l'étude des migrations internationales : le point de vue de l'autonomie comme approche théorique et la notion du projet migratoire comme approche épistémologique. Il ne tente pas de donner une définition de ces notions, mais essaie d'en retracer la généalogie et en particulier de montrer leurs évolutions à travers les objets de recherche qui ont préoccupé leur principal penseur et auteur, Emmanuel Ma Mung, entre les années 1970 et aujourd'hui. Durant cette période, le géographe a élaboré la notion d'autonomie à travers deux grands axes de recherche : la diaspora chinoise et l'entreprenariat ethnique. Depuis 1989, l'auteur est membre de l'équipe du laboratoire Migrinter, dont il a assuré sa direction entre 1995 et 2004

\section{INDEX}

Mots-clés : concept, autonomie, projet migratoire, méthodologie, biographie

\section{AUTEUR}

\section{IRIS POLYZOS}

Docteure en Sociologie urbaine, Université de Poitiers (Migrinter) et Université Technique Nationale d'Athènes. Membre scientifique de l'École française d'Athènes (EFA)

iris.polyzos@efa.gr 